\title{
Nonlinear Optimal Control of Two-Level Large-Scale Systems; Part I - Interaction Prediction Principle
}

\author{
N. SADATI and A.R. MOMENI \\ Intelligent Systems Laboratory, \\ Electrical Engineering Department, \\ Sharif University of Technology, \\ P.O. Box 11365-9363, Tehran, Iran. \\ e-mail:sadati@sina.sharif.edu
}

\begin{abstract}
In this paper, which consists of two parts, a new two-level computational algorithm is used for nonlinear optimal control of large-scale systems. The two-level optimizer uses a new coordination strategy which is based on the gradient of interaction errors instead of the gradient of overall performance function. The advantages of the new method can be categorized into two parts: First, the new formulation is applicable to a large class of problems whilst the classical model coordination method is not. Second, it extremely reduces the number of iterations required for obtaining the overall optimal solution. Although the computational burden of the algorithm in the first level increases slightly, it is shown that there is a great saving in computation time of the overall problem. To demonstrate the significance of the theoretical developments and the computational requirements for new algorithm, a numerical example is presented.
\end{abstract}

\section{INTRODUCTION}

In optimal control of large-scale systems, the control calculations can not be done in a centralized manner, because of high complexity in calculations and dimensionality problem of centralized controller. In these systems, a two- level method can be used for solving the optimal control problems.

For optimization of large-scale systems two coordination principles have been introduced by [1] and [2]. These two coordination methods are named as Interaction Prediction and Interaction Balance Principles. In using these principles a large-scale system is decomposed into several interconnected subsystems and the optimization problem redefines for each subsystem. Then, each subsystem solves its own problem and a coordinator uses one of these principles to coordinate the sub-systems in a manner that the optimal solution for the overall problem can be achieved.

As it is noted, this paper is divided into two parts; in Part I, the Model Coordination of large-scale systems using Interaction Prediction Principle is introduced and in Part II, the Goal Coordination based on Interaction Balance Principle is addressed.

In part I of this paper, by applying the Interaction Prediction Principle, the coordinator sets the prediction values for interaction signals, compares them by their real values and then provides new prediction for interaction signals to reduce the coordination errors to zero.

It should be noted that the classical Model Coordination method, based on Interaction Prediction Principle which is also called feasible method, is usually inapplicable to a large class of problems since it requires that the dimension of local inputs exceeds the number of interaction variables in each subsystem [3],[4].

In the sequel, different sections of part I of this paper are briefly described. A brief description of Model Coordination is given in section II. In section III, the overall optimization problem is decomposed into several subproblems. In section IV, the first-level optimizations are done for nonlinear dynamics using gradient method. In section $\mathrm{V}$, a new gradient based algorithm for coordination of subsystems is described that solves the overall problem in a few number of iterations. Finally, the simulation results are shown followed by concluding remarks in sections VI and VII, respectively.

\section{MODEL COORDINATION AND INTERACTION PREDICTION PRINCIPLE}

Since the Model Coordination based on Interaction Prediction Principle is used in Part I of this paper, this principle is introduced in sequel.

Assume $Z_{i}$ as the set for interaction signals in the $i$ th subsystem and define: $Z=Z_{1} \times Z_{2} \times \cdots \times Z_{N}$. For every $\alpha=\left(\alpha_{1}, \alpha_{2}, \cdots, \alpha_{n}\right)$ in $Z$, let define each subsystem as given in equation (1)

$$
P_{i \alpha}\left(u_{i}\right)=P_{i}\left(u_{i}, \alpha_{i}\right)
$$

where in the above equation, $\alpha$ is the coordination parameter. Now, the $i$ th control problem in the first level is to find $\hat{u}_{i}$ in $U_{i}$ such that

$$
g_{i}\left(\hat{u}_{i}, \alpha_{i}\right)=\min _{u_{i} \in U_{i}} g_{i}\left(u_{i}, \alpha_{i}\right)
$$

Minimization is only over $U_{i}$.

Suppose that $\hat{u}_{1}, \ldots, \hat{u}_{N}$ are the local optimal control inputs for all subsystems and $z_{1}(\alpha), \ldots, z_{N}(\alpha)$ are the interaction signals that occur if the control $\hat{u}_{1}, \ldots, \hat{u}_{N}$ is implemented. The overall optimum is then achieved if the actual interaction signals are precisely equal to the values predicted by the second-level coordinator (Interaction Prediction Principle) [1].

$$
z_{i}(\alpha)=\alpha_{i}
$$

If the Interaction Prediction Principle applies, the 
supremal control action is to find $\alpha$ in $Z$ such that interaction errors $\varepsilon_{i}=\alpha_{i}-z_{i}(\alpha)$ become zero for all subsystems.

Fig. 1 shows the application of Interaction Prediction Principle for coordination of two subsystems.

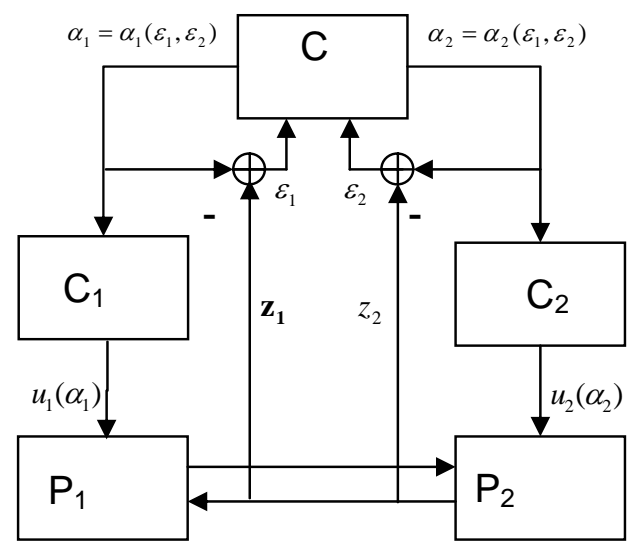

Fig. 1 Application of Interaction Prediction Principle for coordiantion of two subsystems

Now, we need to decompose the overall optimization problem into several sub-problems and then coordinate them in such a way that the overall solution is optimal.

Suppose we have a general nonlinear system described by the following state space equation.

$$
\underline{x}(k+1)=\underline{f}(\underline{x}(k), \underline{u}(k))
$$

where $\underline{x}$ is the state vector, $\underline{u}$ is the control input vector and $f$ is a continuously double differentiable analytical vector function.

The problem is to find $\underline{u}$ which minimizes the cost function given by

$$
J=G_{n+1}(\underline{x}(n+1))+\sum_{k=0}^{n} G_{k}(\underline{x}(k), \underline{u}(k))
$$

where $G_{k}$ is a general nonlinear scalar function of its arguments.

\section{DECOMPOSITION OF THE OVERALL PROBLEM}

Let assume that the overall system is a combination of $N$ interconnected subsystems and each subsystem has a state space equation as follows

$$
\begin{aligned}
& \underline{x}_{i}(k+1)=\underline{f}_{i}\left(\underline{x}_{i}(k), \underline{u}_{i}(k), \underline{z}_{i}(k)\right) \\
& \underline{x}_{i}(0)=\underline{x}_{i}
\end{aligned}
$$

where $\underline{x}_{i}$ is the state vector, $\underline{u}_{i}$ the input and $\underline{z}_{i}$ is the interaction input of the $i$ th subsystem that is assumed to be a nonlinear function of the overall system's state vector.

$$
\underline{z}_{i}(k)=\underline{H}_{i}(\underline{x}(k))
$$

Suppose that overall objective function (5) can be written as the summation of $N$ separated local objective functions, given by.

$$
\begin{aligned}
& J=\sum_{i=1}^{N} J_{i} \\
& J_{i}=G_{i_{n+1}}\left(\underline{x}_{i}(n+1)\right)+\sum_{k=0}^{n} G_{i_{k}}\left(\underline{x}_{i}(k), \underline{u}_{i}(k)\right)
\end{aligned}
$$

Then in each subsystem the problem, is to solve the following subproblems with known $\underline{Z_{p}}$ (A prediction value for $\underline{Z}$ ) coming from the higher level.

$$
\begin{array}{ll}
\min _{\underline{x}_{i}, \underline{u}_{i}} & G_{i_{n+1}}\left(\underline{x}_{i}(n+1)\right)+\sum_{k=0}^{n} G_{i_{k}}\left(\underline{x}_{i}(k), \underline{u}_{i}(k)\right) \\
\text { s.t. } & \underline{x}_{i}(k+1)=\underline{F}_{i}\left(\underline{x}_{i}(k), \underline{u}_{i}(k), \underline{z}_{\underline{p}}(k)\right) \\
& \underline{x}_{i}(0)=\underline{x}_{0}
\end{array}
$$

while in the second level, the problem is to update the coordination parameter $\underline{Z}$, such that the interaction errors become zero [2].

\section{FIRST-LEVEL OPTIMIZATION}

In the first level the problem is to solve the optimization problems described in (9), by assuming known constant values of $Z_{p}$ from the second level. So by adding the following terms

$$
\underline{\lambda}_{i}^{T}(k)\left[\underline{x}_{i}(k+1)-\underline{f}_{i}\left(\underline{x}_{i}(k), \underline{u}_{i}(k), \underline{z}_{p}(k)\right)\right]
$$

the Lagrangian can be written as

$$
\begin{aligned}
L_{i} & =G_{i_{n+1}}\left(\underline{x}_{i}(n+1)\right)+\sum_{k=0}^{n} G_{i_{k}}\left(\underline{x}_{i}(k), \underline{u}_{i}(k)\right) \\
& +\sum_{k=0}^{n} \underline{\lambda}_{i}^{T}(k)\left[\underline{x}_{i}(k+1)-\underline{f}_{i}\left(\underline{x}_{i}(k), \underline{u}_{i}(k), \underline{z}_{i}(k)\right)\right]
\end{aligned}
$$

To solve this problem, an algorithm based on optimality necessary conditions can be used as below [5]:

1. Choose initial values for $\underline{u}_{i}(0)$ to $\underline{u}_{i}(n)$.

2. Use ${\underline{x_{0}}}_{i}$ and values for $\underline{u}_{i}(k)$ and $\underline{z}_{\underline{p}}(k)$ to compute the values of $\underline{x}_{i}(1)$ to $\underline{x}_{i}(n+1)$, using state space equations of the system.

3. Calculate $\underline{\lambda}_{i}(k)$ for $k=n, n-1, \ldots, 0$ backward in time, by using the following necessary conditions:

$$
\begin{aligned}
& \underline{\lambda}_{i}(n)=-\frac{\partial G_{i_{n+1}}\left(\underline{x}_{i}(n+1)\right)}{\partial \underline{x}_{i}(n+1)} \\
& \underline{\lambda}_{i}(k-1)=-\frac{\partial G_{i_{k}}\left(\underline{x}_{i}(k), \underline{u}_{i}(k)\right)}{\partial \underline{x}_{i}(k)} \\
& +\left(\frac{\partial \underline{f}_{i}\left(\underline{x}_{i}(k), \underline{u}_{i}(k), \underline{z}_{p_{i}}(k)\right)}{\partial \underline{x}_{i}(k)}\right)^{T} \underline{\lambda}_{i}(k) \\
& ; k=n, n-1, \cdots, 0
\end{aligned}
$$


4. Calculate $\frac{\partial L_{i}}{\partial \underline{u}_{i}(k)}$ using $\underline{x}_{i}(k)$ and $\underline{\lambda}_{i}(k)$.

5. Update $\underline{u}_{i}(k)$ by the following relation:

$\underline{u}_{i}^{(l+1)}(k)=\underline{u}_{i}^{(l)}(k)-\rho \frac{\partial L_{i}}{\partial \underline{u}_{i}(k)}$

where $\rho>0$.

6. If $\sum_{k=0}^{n}\left\|\frac{\partial L_{i}}{\partial \underline{u}_{i}(k)}\right\|^{2}<\varepsilon$ stop the algorithm, else go to step (2).

\section{COORDINATION USING THE GRADIENT OF ERRORS}

In the second level, the goal is to update $Z_{p}$ in order to decrease the interaction errors defined as

$$
\underline{e}_{i}(k)=\underline{z}_{i}(k)-\underline{z}_{\underline{p}_{i}}(k)
$$

where $\underline{Z}_{i}(k)$ is the real value of $i$ th subsystem interaction signal coming from (7) if $\underline{x}(k)$ is local optimal value of the overall system's state vector obtained from first-level calculations based on prediction $Z_{p}$.

In the classical method of coordination, the gradient of overall performance function was used for coordination [4], [6]. In this paper, the gradient of interaction errors is used for coordination. This method first proposed by Sadati [7]-[9], has faster convergence rate than the previous ones.

Since the gradient of interaction error is used for coordination, the gradient of interaction errors related to the coordination parameters need to be found.

Let us define the following vectors for convenience:

$$
\begin{array}{cc}
\underline{e}=\left[\begin{array}{c}
\underline{e}_{1} \\
\underline{e}_{2} \\
\vdots \\
\underline{e}_{N}
\end{array}\right] & \underline{\Lambda}_{i}=\left[\begin{array}{c}
\underline{\lambda}_{i}(0) \\
\underline{\lambda}_{i}(1) \\
\vdots \\
\underline{\lambda}_{i}(n)
\end{array}\right] \\
\underline{Z}_{i}=\left[\begin{array}{c}
\underline{Z}_{i}(0) \\
\underline{z}_{i}(1) \\
\vdots \\
\underline{z}_{i}(n)
\end{array}\right] \quad, \quad \underline{Z}=\left[\begin{array}{c}
\underline{Z}_{1} \\
\underline{Z}_{2} \\
\vdots \\
\underline{Z}_{N}
\end{array}\right], \quad \underline{Z}_{p}=\left[\begin{array}{c}
\underline{Z}_{p_{1}} \\
\frac{Z_{p}}{亠_{2}} \\
Z_{Z_{N}}
\end{array}\right]
\end{array}
$$

The sum-squared error is also given by:

$$
S E=\frac{1}{2} \underline{e}^{T} \underline{e}
$$

Now, to update $Z_{p}$, we need to move along the direction of the vector $\frac{\partial S E}{\partial Z_{p}}$ in each iteration. Therefore

$$
{\underline{Z_{p}}}^{(l+1)}=\underline{Z_{p}^{(l)}}-\eta \frac{\partial S E}{\partial \underline{Z_{p}}}
$$

where $\eta$ is the step length and $l$ is the iteration index.

Since we have

$$
\frac{\partial S E}{\partial \underline{Z_{p}}}=\left(\frac{\partial \underline{\underline{e}}}{\partial \underline{Z_{p}}}\right)^{T} \underline{e} \underline{\underline{\Delta}} P^{T} \underline{e}
$$

Therefore, we need to compute $\frac{\partial \underline{e}}{\partial \underline{Z_{p}}}$ So we have

$$
P=\frac{\partial \underline{e}}{\partial \underline{Z_{p}}}=\frac{\partial \underline{Z}}{\partial \underline{Z_{p}}}-\frac{\partial Z_{p}}{\partial \underline{Z_{p}}} \triangleq Q-I
$$

where $\mathbf{I}$ is an identity matrix with appropriate dimension.

Now using chain rule, $Q$ can be written as follows

$$
Q=\frac{\partial \underline{Z}}{\partial \underline{Z_{p}}}=\frac{\partial \underline{Z}}{\partial \underline{X}} \times \frac{\partial \underline{X}}{\partial \underline{Z_{p}}} \underline{\underline{\Delta}} E \times D
$$

Considering the interaction relation between subsystems, $E$ can be computed easily from (19a) and (19b).

$$
\begin{aligned}
& E=\left[\begin{array}{cccc}
E_{11} & E_{12} & \cdots & E_{1 N} \\
E_{21} & E_{22} & \cdots & E_{2 N} \\
\vdots & \vdots & & \vdots \\
E_{N 1} & E_{N 2} & \cdots & E_{N N}
\end{array}\right] \\
& E_{i j}=\left[\begin{array}{cccc}
\frac{\partial \underline{H}_{i_{0}}}{\partial \underline{x}_{j}(0)} & & 0 & \\
& \frac{\partial \underline{H}_{i_{1}}}{\partial \underline{x}_{j}(1)} & & \\
& 0 & & \frac{\partial \underline{H}_{i_{n+1}}}{\partial \underline{x}_{j}(n+1)}
\end{array}\right]
\end{aligned}
$$

Since optimum local values of the $i$ th subsystem is just related to the value of $\underline{Z}_{p_{i}}$; not $\underline{Z_{p}}$ j for $j \neq i$, the matrix

$D$ will be block diagonal [9].

$$
D=\text { block diagonal }\left(D_{i}\right)
$$

where

$$
D_{i}=\frac{\partial \underline{X}_{i}}{\partial \underline{Z}_{p}}
$$

Let $\underline{x}_{i}(k), \underline{u}_{i}(k)$ and $\underline{\lambda}_{i}(k)$ be the optimum values provided by the first level using kown $Z_{p}$. These values satisfy the necessary optimality conditions at the first level. i.e.

$$
\frac{\partial L_{i}}{\partial \underline{x}_{i}}=\underline{0}, \frac{\partial L_{i}}{\partial \underline{u}_{i}}=\underline{0}, \frac{\partial L_{i}}{\partial \underline{\lambda}_{i}}=\underline{0}
$$

The necessary conditions in (22) can now be written in a compact form, by the following equation

$$
\frac{\partial L_{i}\left(\underline{W}_{i}, \underline{Z_{p}} i\right)}{\partial \underline{W}_{i}}=\underline{0}
$$

where

$$
\underline{W}_{i} \Delta\left[\begin{array}{l}
\underline{U}_{i} \\
\underline{X}_{i} \\
\underline{\Lambda}_{i}
\end{array}\right]
$$


Now, by considering of small variations in $\underline{W_{i}}$ and $\underline{Z_{p}} i$, the following equation results from (23):

$$
\frac{\partial^{2} L_{i}}{\partial \underline{W}_{i} \partial \underline{W}_{i}} \delta \underline{W}_{i}+\frac{\partial^{2} L_{i}}{\partial \underline{Z}_{\underline{p}_{i}} \partial \underline{W}_{i}} \delta \underline{Z}_{\underline{p}_{i}}=\underline{0}
$$

If $\delta \underline{Z}_{\underline{p}_{i}}$ and $\delta \underline{W}_{i}$ become very close to zero, them $\frac{\partial \underline{W}_{i}}{\partial \underline{Z}_{p_{i}}}$ can be written as bellow

$$
\frac{\partial \underline{W}_{i}}{\partial \underline{Z}_{p}}=-\left[\frac{\partial^{2} L_{i}}{\partial \underline{W}_{i} \partial \underline{W}_{i}}\right]^{-1} \frac{\partial^{2} L_{i}}{\partial \underline{Z}_{p} \partial \underline{W}_{i}}
$$

On the other hand, we have

$$
\underline{X}_{i}=\left[\begin{array}{lll}
\mathbf{0} & \mathbf{I} & \mathbf{0}
\end{array}\right] \underline{W}_{i} \underline{\underline{\Delta}} S \underline{W}_{i}
$$

Therefore, $D_{i}$ can be computed from the following equation:

$$
D_{i}=\frac{\partial \underline{X}_{i}}{\partial \underline{Z}_{p_{i}}}=-S\left[\frac{\partial^{2} L_{i}}{\partial \underline{W}_{i} \partial \underline{W}_{i}}\right]^{-1} \frac{\partial^{2} L_{i}}{\partial \underline{Z}_{\underline{p}_{i}} \partial \underline{W}_{i}}
$$

Now by using the following algorithm, Optimization of large-scale dynamic systems using Interaction Prediction Principle and the new gradient-based coordination is possible.

1. Start with initial values for coordination parameters.

2. Solve the first level optimization problems with the known coordination parameters using gradient method and calculate $\underline{x}_{i}, \underline{u}_{i}, \underline{\lambda}_{i}$ and matrix $D_{i}$ using (28).

3. Calculate the interaction errors and the gradient matrix by (13) and (17) - (20), respectively

4. Update $Z_{p}$ using (15) and (16).

5. Calculate the sum-squared errors. If it is smaller than a desired value terminate the algorithm, else go to step (2)

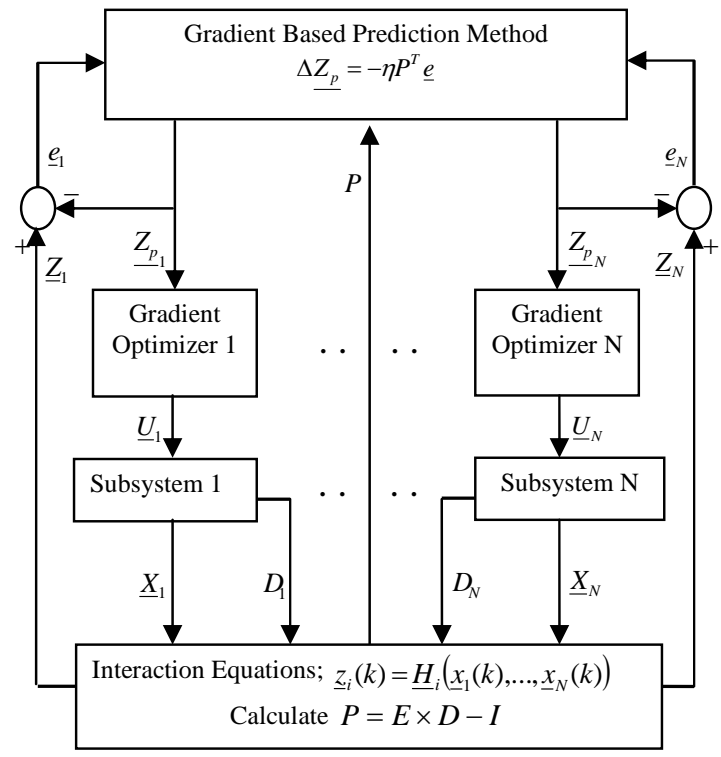

Fig. 2 Two-level control using the new coordination strategy

\section{SIMULATION RESULTS}

As a system for simulation, a system composed of 4 connected water tanks is chosen [10]. This system is shown in Fig. 3.

Choosing $\left[\begin{array}{ll}h_{1} & h_{3}\end{array}\right]^{T}$ as $\underline{h}_{1}$ and $\left[\begin{array}{ll}h_{2} & h_{4}\end{array}\right]^{T}$ as $\underline{h}_{2}$, the discrete time state space equations for this system can be given as

$$
\begin{aligned}
& \underline{h}_{1}(k+1)=\underline{h}_{1}(k)+A_{1} \sqrt{\underline{h}_{1}(k)}+B_{1} u_{1}(k)+D_{1} z_{1}(k) \\
& \underline{h}_{2}(k+1)=\underline{h}_{2}(k)+A_{2} \sqrt{\underline{h}_{2}(k)}+B_{2} u_{2}(k)+D_{2} z_{2}(k)
\end{aligned}
$$

where the unknown parameters are listed bellow

$$
\begin{aligned}
& z_{1}(k)=r_{4,1} \frac{s_{2,4} T}{s_{1,1}} \sqrt{2 g h_{4}(k)} \quad z_{2}(k)=r_{3,2} \frac{s_{2,3} T}{s_{1,2}} \sqrt{2 g h_{3}(k)} \\
& A_{1}=\left[\begin{array}{cc}
-\frac{S_{2,1} T}{S_{1,1}} \sqrt{2 g} & r_{3,1} \frac{S_{2,3} T}{S_{1,1}} \sqrt{2 g} \\
0 & -\frac{S_{2,3} T}{S_{1,3}} \sqrt{2 g}
\end{array}\right] \\
& A_{2}=\left[\begin{array}{cc}
-\frac{S_{2,2} T}{S_{1,2}} \sqrt{2 g} & r_{4,2} \frac{S_{2,4} T}{S_{1,2}} \sqrt{2 g} \\
0 & -\frac{S_{2,4} T}{S_{1,4}} \sqrt{2 g}
\end{array}\right] \\
& B_{1}=\left[\begin{array}{c}
0 \\
\frac{T}{S_{1,2}}
\end{array}\right], B_{2}=\left[\begin{array}{c}
0 \\
\frac{T}{S_{1,4}}
\end{array}\right], D_{1}=\left[\begin{array}{l}
1 \\
0
\end{array}\right], D_{2}=\left[\begin{array}{l}
1 \\
0
\end{array}\right] \\
& {\left[\begin{array}{ll}
r_{3,1} & r_{3,2} \\
r_{4,1} & r_{4,2}
\end{array}\right]=\left[\begin{array}{ll}
0.8 & 0.2 \\
0.2 & 0.8
\end{array}\right]} \\
& S=\left[\begin{array}{llll}
10^{-3} & 10^{-3} & 10^{-3} & 10^{-3} \\
10^{-6} & 10^{-6} & 10^{-6} & 10^{-6}
\end{array}\right], T=50 \mathrm{sec}
\end{aligned}
$$

Now, the overall objective function can be written as

$$
\begin{array}{r}
J=\frac{1}{2}\left\|\underline{h}_{1}(n+1)-\underline{h}_{1 f}\right\|_{Q_{1}}^{2}+\frac{1}{2}\left\|\underline{h}_{2}(n+1)-\underline{h}_{2 f}\right\|_{Q_{2}}^{2} \\
+\sum_{k=0}^{n}\left[\frac{1}{2}\left\|\underline{h}_{1}(k)-\underline{h}_{1 f}\right\|_{Q_{1}}^{2}+\frac{1}{2}\left\|u_{1}(k)-u_{1 f}\right\|_{R_{1}}^{2}\right] \\
+\sum_{k=0}^{n}\left[\frac{1}{2}\left\|\underline{h}_{2}(k)-\underline{h}_{2 f}\right\|_{Q_{2}}^{2}+\frac{1}{2}\left\|u_{2}(k)-u_{2 f}\right\|_{R_{2}}^{2}\right] \\
h_{2}
\end{array}
$$

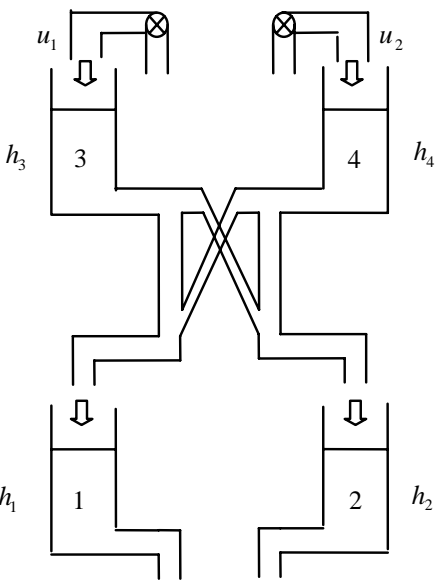

Fig. 3 Connected 4 Water Tanks 
where

$$
\begin{aligned}
& Q_{1}=Q_{2}=\left[\begin{array}{ll}
1 & 0 \\
0 & 1
\end{array}\right], \quad R_{1}=R_{2}=10^{11}, \quad n=20 \\
& \underline{h}_{1 f}=\underline{h}_{2 f}=\left[\begin{array}{l}
2 \\
2
\end{array}\right] \quad, \quad u_{1 f}=u_{2 f}=6.264 \times 10^{-6}
\end{aligned}
$$

and the initial states for each subsystem are chosen to be

$$
\underline{h_{0}}=\left[\begin{array}{ll}
2.2797 & 2.5
\end{array}\right]^{T} \quad \underline{h_{0}}=\left[\begin{array}{ll}
1.6797 & 1.5
\end{array}\right]^{T}
$$

The optimization problem for system of connected 4 liquid tanks was solved on an IBM Pentium III $550 \mathrm{MHZ}$ digital computer at ISL using feasible method and the proposed algorithm. Using the first method, convergence to the optimum took place in 23 iterations which required 240 ms time to execute each iteration (Fig. 4(b) and Table II). But for the latter, the convergence to the optimum took place in 6 iterations which required $320 \mathrm{~ms}$ time to execute for each iteration (Fig. 4(a) and Table I). Therefore, the total computational times are $5.5 \mathrm{sec}$ and $1.9 \mathrm{sec}$, respectively. It means up to $65 \%$ time saving is occurred. It is instructive to mention that the coordination error criteria are different for two approaches.

Fig. 5 shows the optimal inputs, calculated for this system. In Fig. 6, optimal state responses of the system are shown. If the centralized optimization is done, the global solution is found to be very close to the solution obtained using the proposed approach.

Table I Required time of execution for each iteration (new approach).

\begin{tabular}{|c|c|c|c|}
\cline { 2 - 4 } \multicolumn{1}{c|}{} & $\begin{array}{c}\text { First-level } \\
\text { optimization }\end{array}$ & $\begin{array}{c}\text { Computing } \\
\mathrm{D}_{\mathrm{i}}\end{array}$ & $\begin{array}{c}\text { Second level } \\
\text { computations }\end{array}$ \\
\hline $\begin{array}{c}\text { Computational } \\
\text { time (ms) }\end{array}$ & 250 & 60 & 10 \\
\hline
\end{tabular}

Table II Required time of execution for each iteration in detail (classical method).

\begin{tabular}{|c|c|c|}
\cline { 2 - 3 } \multicolumn{1}{c|}{} & $\begin{array}{c}\text { First-level } \\
\text { optimization }\end{array}$ & $\begin{array}{c}\text { Second level } \\
\text { computations }\end{array}$ \\
\hline $\begin{array}{c}\text { Computational } \\
\text { time (ms) }\end{array}$ & 230 & 10 \\
\hline
\end{tabular}

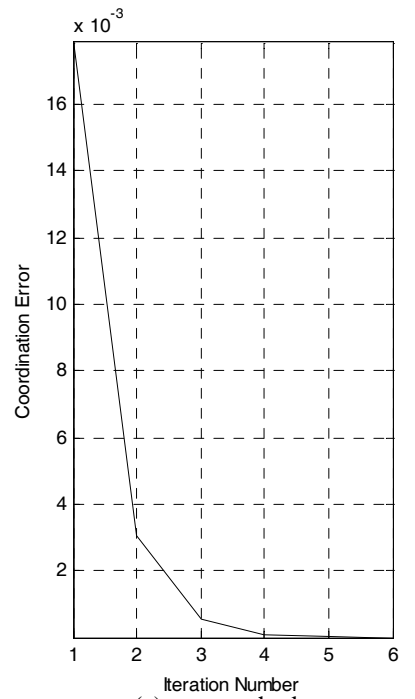

(a) new method

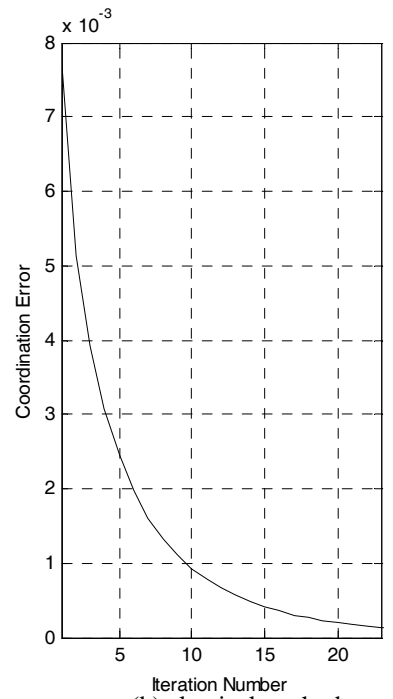

(b) classical method

Fig 4. Comparison of errors using the new and the classical coordination approaches
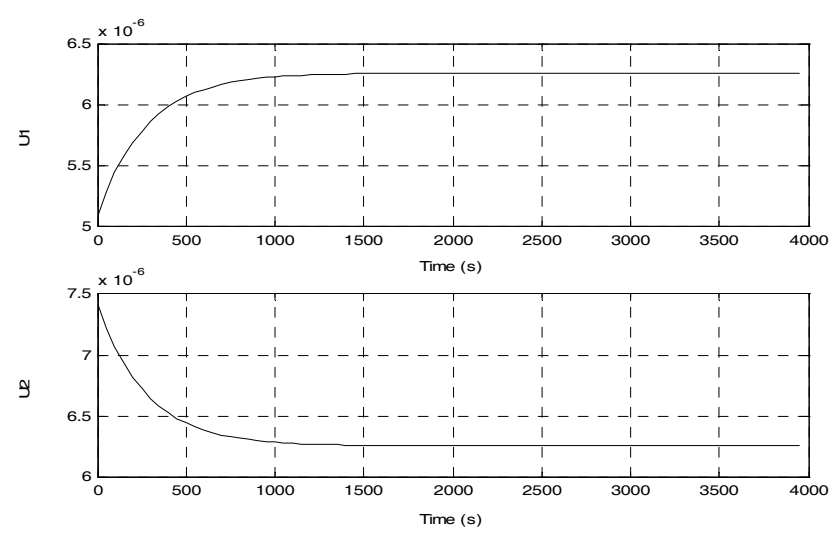

Fig. 5 Optimal inputs for each subsystem 

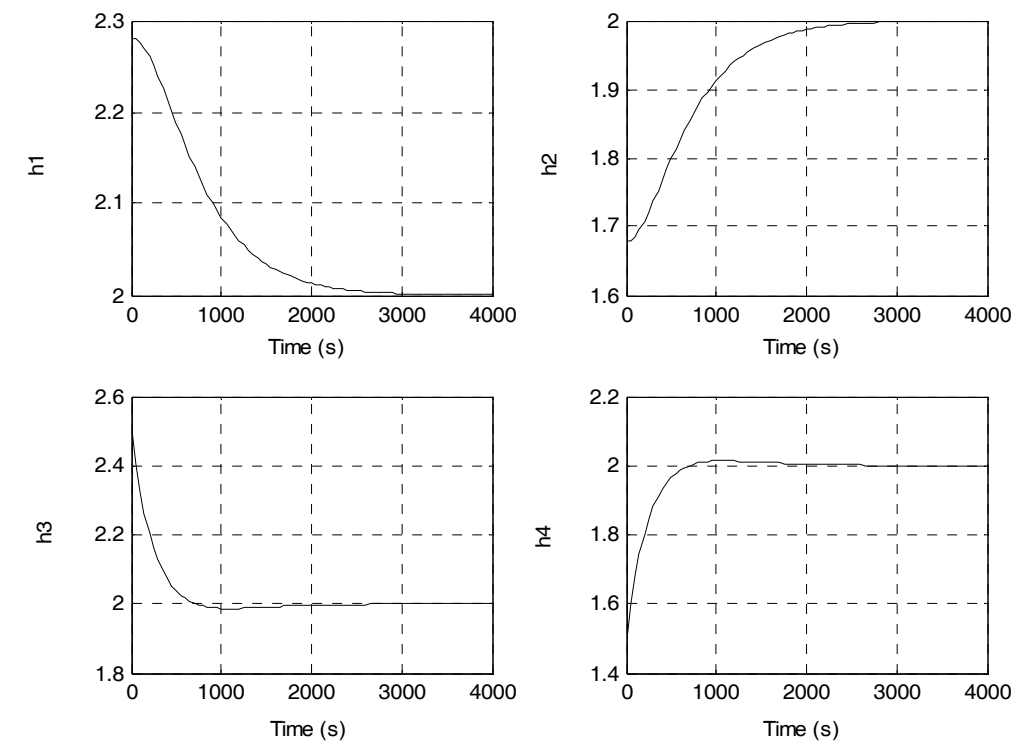

Fig. 6 Optimal state responses using the new model coordination approach

\section{CONCLUSION}

In this part of the paper, a new two-level method for nonlinear optimization of large-scale systems is proposed. This two-level algorithm is based on Interaction Prediction Principle. In the second level, a new coordinator based on the gradient of interaction errors is used. The new methodology makes the number of information exchange between two levels reduce considerably in compare to the classical method. The numerical simulation demonstrates that the computational time using the new approach decreases substantially.

As mentioned before, the classical method is not applicable to a large class of problems while the proposed approach gives us the ability to use Interaction Prediction Principle for solving optimization problems in two-level form without imposing any additional restrictions.

To compare this work with the mixed method [2] which can be viewed as a hierarchical methodology based on Interaction Prediction Principle, it is perceived that the dimension of coordination variables is decreased using the new approach. On the other hand, when we are going to use the mixed method, two values should be chosen for the learning factor instead of one and it is quite evident that the best fitting of these parameters will need more efforts.

The Goal Coordination of this approach is also the subject of Part II of this paper.

\section{REFERENCES}

[1] M.D. Mesarovic, D. Macko and Y. Takahara, "Two coordination principles and their applications in large-scale systems control,’Proc IFAC Congr., Warsaw, Poland, 1969.

[2] M.D. Mesarovic, D. Macko and Y. Takahara, Theory of Hierarchical Multilevel Systems. New York: Academic Press, 1970.

[3] N.R. Sandell, P. Varaiya, M. Athans and M. G. Safonov "survey of decentralized control methods for large scale
Systems”, IEEE Trans. on Automatic Control, vol. 23, no. 2, April 1978, PP. 108-128.

[4] M. Jamshidi, Large-Scale Systems: Modeling, Control and Fuzzy Logic. Prentice Hall, 1997.

[5] M.G. Singh and A. Titli, Systems: Decomposition, Optimization and Control. Oxford: Pergamon Press, 1978.

[6] M.D. Singh, Dynamical Hierarchical Control. Rev. Ed., Amsterdam, North Holland, 1980.

[7] N. Sadati, "A gradient based method for model coordination of large-scale systems," Sharif University of Technology, Electrical Engineering Department, Report no. SUT-ICSL (18), 1997.

[8] N. Sadati, “A new two-level gradient based approach for intelligent coordination of large-scale systems; Part I Interaction prediction principle," Sharif University of Technology, Electrical Engineering Department, Report no. SUT-ICSL (30), 2000.

[9] N. Sadati, "A novel approach to coordination of large-scale systems; Part I - Interaction prediction principle,” in press, IEEE International Conference of Industrial Technology, Hong Kong, Dec. 2005.

[10] J.A. Roubos, S. Mollov, R. Babuska and H.B. Vertruggen, "Fuzzy model-based predictive control using Takagi-Sugeno models”, Int. J. Approx. Reason, vol. 22, 1999, pp. 3-30. 